\title{
An Improved Weighted Moving Average Methods Based on Transferring Weights for an Analytical Process Data
}

\author{
Ruishan $\mathrm{Du}^{*}$ and Hui Yang
}

School of Computer and Information Technology, Northeast Petroleum University, Daqing 163318, P.R. China

\begin{abstract}
Time series forecasting is an active research area that has drawn considerable attention for applications in a variety of areas. Moving Average is one of widely known technical indicator used to predict the future data in time series analysis. During its' development, many variation and implementation have been made by researchers. One of its' widely used variation is Weighted Moving Average that gives a special weighting to more recent data than the older data, which could not be found in Simple Moving Average method. This paper aims to introduce a new approach of moving average method in time series analysis. The approach will propose transferring weights mothod as the new weighting factor in view of the lagging and incomplete problems of the weighted moving average method. Both theoretical and empirical findings have suggested that the proposed method can be an effective method of improving upon their predictive performance, especially when the models in improving the lagging and Outburst value. In this paper, the models are implemented in order to overcome data limitations of weighted moving average models, thus obtaining more accurate results. Experimental results of Water Injection in oil field indicate that the models exhibit effectively improved forecasting accuracy so that the model proposed can be used as an alternative to forecasting tools. The result of the proposed method shows a promising result in this preliminary work.
\end{abstract}

Keywords: Time series, transferring weights, outburst value, weighted moving average.

\section{INTRODUCTION}

Moving average model is the basic tools and basic concepts of time series analysis, it was born from the index scale series used in banks in 1797-1819, when banks use it because of it reveal wealth of bank, at the same time cover the true data [1], it is a simulation tool for trend, in essence, it is a derived sequence. Therefore, no doubt that moving average curve followed the trend of the raw data, and effectively weakened the influence due to staff errors, exceptional circumstances of original data, which played the role of smoothing, from methodological classification, moving average is a smoothing technique. Moving average has been widely used based on the timing of forecase, provide realtime decision support for practical applications. In recent years, the improvements of moving average model and the combined with other theory become a research hotspot of data preprocessing [2-12].

Moving average method is a method raised for moving average lag issues, this principle can be directly calculated correction factor based formula, easy to calculate and summarize, the present method is based on a simple moving average further amendments [4], did not analysis lag exist on the weighted moving average, Literature [5] proposed moving average filtering algorithm, and its essence is based on an average of dynamic correction obtained the data phase, to eliminate the lag. The essence of the method is also the improvement of simple moving average.

\section{THE PRINCIPLE OF MOVING AVERAGE}

\subsection{Moving Average Value}

Moving average is a sequence of $n$ items of time to calculate the average of a sequence a plurality of consecutive items. The first of a sequence of consecutive $m$ item the first item is the first item in the original $m$ sequence; second consecutive sequence of the first $n$ items of the original of the second $m$ sequence; ......; the last sequence of $n$ items the first term of the sequence of $n-m+1$ entries.

\subsection{Simple of Moving Average}

Simple moving average is the degree of influence at alltime points considered equal, so the weight of the elements are equal. Simple moving average model is calculated as follows:

$$
F_{n}=\left(\sum_{i=1}^{n} \varepsilon_{i}\right) / n
$$

$F_{n}$ is a sliding average of the $n ; n$ is moving step; $\varepsilon_{i}$ is the pre $i$ actual values of $F_{n}$. 


\subsection{Weighted of Moving Average}

Weighted moving average model is based on different time point has different predicted during a sliding to distribute varying weight, since the more recent data has greater impact on the predicted values, usually given move big weight to recent data, less weight to longer data, the sum of weight aggregate is 1 . Weighted moving average is calculated as follows, $\omega_{\mathrm{i}}$ is weight:

$F_{n}=\sum_{i=1}^{n} w_{i} \varepsilon_{i}, \sum_{i=0}^{n} w_{i}=1$

\section{IMPROVING THE LAG AND OUTBURST VALUE BASED ON WEIGHT TRANSFER}

Weighted moving average for the existence of the lag and the outburst value, this paper presents a "weight shift" method, which can effectively improve the overall weighted moving average method for smoothing effect. The following are the weights shifted weighted moving average method and on the improvement measures are introduced.

\subsection{Weight Transfer}

Weight transfer is based on the weighted moving average model concerned and it is to transfer the weight of the weighted moving average model weights have been assigned but the actual value does not exist or is empty point in time weight redistributed to the greatest impact on the predicted target point in time.

$$
\begin{aligned}
& F_{n}=w_{1} \varepsilon_{0}+w_{2} \varepsilon_{1}+w_{3} \varepsilon_{2}+\cdots+w_{n+1} \varepsilon_{n} \\
& F_{n}=\left(w_{1}+w_{n+1}\right) \varepsilon_{0}+w_{2} \varepsilon_{1}+w_{3} \varepsilon_{2}+\cdots+w_{n} \varepsilon_{n-1}
\end{aligned}
$$

\subsection{Lag Improvement of Weighted Moving Average}

Common weighted moving average lags, the paper in the literature [6] proposed on the basis of the further lag improved method, is the actual value does not exist case weights transfer method, to ensure the weighted moving average smoothing, smoothing function under the premise of minimizing the impact of lag, and moving step length, the stronger the more obvious hysteresis, hysteresis is also more meaningful improvements.

Based on the existing optimized weighted moving average method to improve an already lagging point weighted moving average model, the specific method is shown as follows:

$$
\begin{aligned}
& F_{t}=\varepsilon_{t},(1 \leq t \leq n-1) \\
& F_{t}=\sum_{i=1}^{t} w_{i} \varepsilon_{i-1},\left(t>n-1, \sum_{i=1}^{t} w_{i}=1\right)
\end{aligned}
$$

The number of lag is $n-1$, which is shown as follows:

$$
F_{t}=\sum_{i=1}^{t} w_{i} \varepsilon_{i-1},\left(1 \leq t \leq n-1, \sum_{i=1}^{t} w_{i}=1\right)
$$

$$
w_{1}+=\sum_{i=t}^{n-1} w_{i+1}
$$

When $t>n-1$, the result as same as formula (6), $w_{1}$ is the weight of $\varepsilon_{0}, w_{2}$ is the weight of $\varepsilon_{1}$, and so on. Improvements the $n-1$ lag time points in accordance with the above method, if the first $n$ of the value does not exist, the result is $w_{1}=w_{1}+w_{n+1}$.

\subsection{Outburst Value Improvement of Weighted Moving Average}

When a large amount of data, it is inevitable there will be data outburst phenomena, and outburst data for predicting results could have a huge impact. This paper presents a weighted moving average hopping on the value of improvements, the actual value is null case weights transfer method that can reduce the maximum value affects the outburst.

Improved method: If the first point in time the actual value is null, the value is the outburst, it will be the first point in time the weight transferred to the predictive value of the greatest impact on the target point in time. The outburst values are not frequently the case, the use of weight shift thinking, reducing the value of the predicted outcome outburst effects, effectively avoiding the outburst to bring the value of the weight loss and weight imbalance effects.

\section{APPLICATION}

With the intensified energy conservation field, focusing on early warning of energy consumption indicators has become particularly important, weighted moving average model predicted early in the curve smoothing played a key role, an oil mine as an example, water system efficiency, and the date the transfer curve using the weight-based weighted moving average model, the next picture shows the mine since January 2008 to May 2012, 53 months of the efficiency of the system based on time series graph, as shown Fig. (1).

\subsection{Determine of Moving Step Length}

Moving step selection is the key weighted moving average model, the results for the smooth movement play a decisive role. Values taken too small, although the model can sensitively reflect the recent trend, but may also reflect the random disturbances over sensitive data obtained as a basis for strong fluctuations, which may cause deviation result; take too large for random time sequence contains low sensitivity to changes, which cannot reflect the recent keen trends. Therefore, in the selection of, must be combined with practical experience. Here, the graph observed time period is 5, there is a short peak time is set to 5 .

\subsection{Determine of Weight}

This application can be understood from the predicted time to a time point closer greater the weight, and the weights sum to 1 , based on experience and comprehensive consideration, the weights are set to: $0.3,0.25,0.2,0.15$, and 0.1 . The weight has been improved into a hysteresis point weighted moving average equation is shown: 


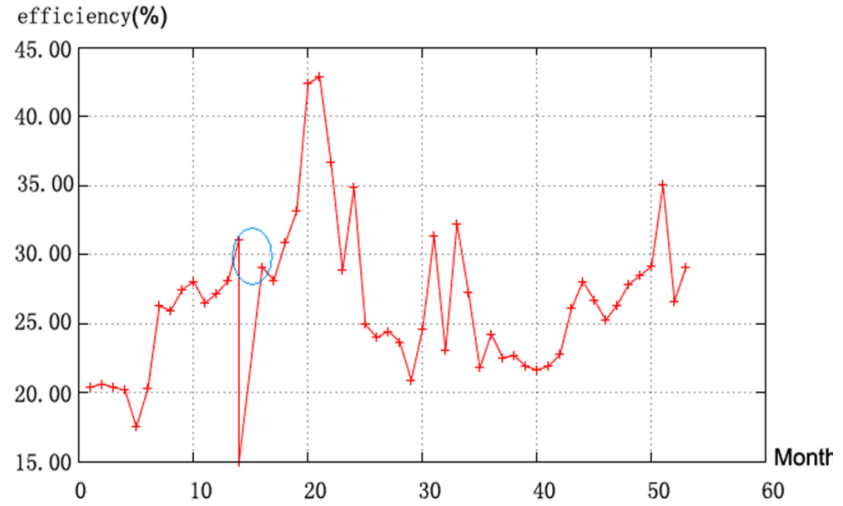

Fig. (1). Water Injection System Efficiency.

$$
F_{5}=0.3 \varepsilon_{0}+0.25 \varepsilon_{1}+0.2 \varepsilon_{2}+0.15 \varepsilon_{3}+0.1 \varepsilon_{4}
$$

\subsection{Improved of Lag in Time Point}

Since moving step is 5 , according to the weighted moving average formula can be commonly found in a five-month lag sliding, weighted moving average of the original formula to improve, this application is based on the four points in time lag, the formula is shown as follows:

$$
F_{t}=\sum_{i=\mathrm{t}}^{4} w_{i} \varepsilon_{i-1}
$$

Improved the lag of weighted moving average is shown as follows:

Step 1 the sliding average of 5 to 53 month:

$F_{5}=w_{1} \varepsilon_{0}+w_{2} \varepsilon_{1}+w_{3} \varepsilon_{2}+w_{4} \varepsilon_{3}+w_{5} \varepsilon_{4}$

Step 2 the sliding average of the first 4 months:

$F_{4}=\left(1-w_{2}-w_{3}-w_{4}\right) \varepsilon_{0}+w_{2} \varepsilon_{1}+w_{3} \varepsilon_{2}+w_{4} \varepsilon_{3}$

Step 3 the sliding average of the first 3 months:

$F_{3}=\left(1-w_{2}-w_{3}\right) \varepsilon_{0}+w_{2} \varepsilon_{1}+w_{3} \varepsilon_{2}$

Step 4 the sliding average of the first 2 months:

$F_{2}=\left(1-w_{2}\right) \varepsilon_{0}+w_{2} \varepsilon_{1}$

Step 5 the sliding average of the first 1 months:

$F_{1}=\varepsilon_{0}$

The first four months according to the existing method of weight transfer method and comparative data shown in Table 1:

Above table shows, the existing improved weighted mov-

Table 1. Comparative of lag transfer method.

\begin{tabular}{|c|c|c|c|}
\hline $\mathbf{z}$ & System efficiency & Existing Improved & $\begin{array}{c}\text { Weight Trans- } \\
\text { fer }\end{array}$ \\
\hline \hline 200801 & 20.39 & 20.59 & 20.59 \\
\hline 200802 & 20.63 & 20.65 & 20.57 \\
\hline 200803 & 20.37 & 20.37 & 20.459 \\
\hline 200804 & 20.17 & 20.17 & 20.345 \\
\hline
\end{tabular}

ing average model results obtained without any change, but the actual worth to take the original, while the weighted based on the weight transfer slip model has played an effective role in smoothing, so that the weighted average thought of the all-time points have played role in ensuring the overall consistency.

\subsection{Improved of Outburst Value}

As shown in Fig. (1), there is an outburst between the two points in blue circle, the outburst is March 2009, because the moving step is 5, the main effect in March to July, five months running average, existing methods and weighting value transfer method hopping process for comparison of the results shown in Table 2 .

According to comparison table, the results of weight transfer closer to the actual value, which effective make up the outburst effect.

\subsection{Analysis of the Results}

Fig. (2) is comparison char, red line shows the original water system efficiency curve, green line shows the improved weighted moving average curve improved and blue line shows the proposed weighted moving average method based on weight transfer. Fig. (3) is an enlarged display of Fig. (2), red line shows the original water system efficiency curve and blue line

shows the proposed weighted moving average method based on weight transfer, through which is shown the effect of smoothing, and it is a strong generalization ability data preprocessing method can be extended to various fields.

Fig. (4) is the enlargement of Fig. (2), it is shown that the 
Table 2. Comparative of outburst transfer method.

\begin{tabular}{|c|c|c|c|}
\hline Time & System efficiency & Existing Improved & $\begin{array}{c}\text { Weight Trans- } \\
\text { fer }\end{array}$ \\
\hline \hline 200903 & Jump value & 20.12 & 29.45 \\
\hline 200904 & 29.10 & 21.8815 & 29.1565 \\
\hline 200905 & 28.13 & 23.188 & 28.814 \\
\hline 200906 & 30.85 & 25.2175 & 29.845 \\
\hline 200907 & 33.14 & 27.6455 & 30.9595 \\
\hline
\end{tabular}

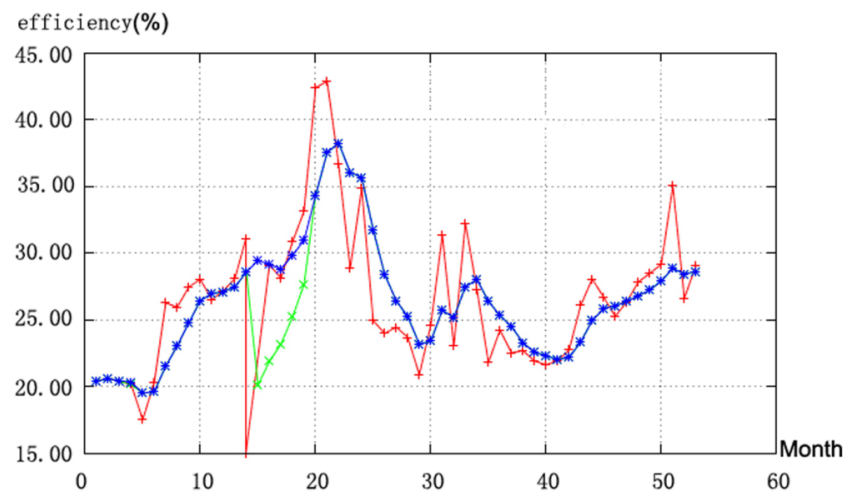

Fig. (2). Three kinds of water injection curve.

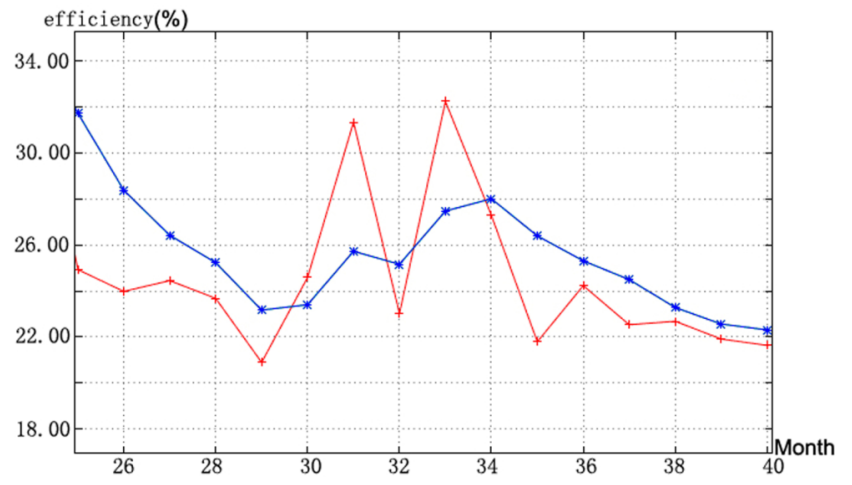

Fig. (3). Curve smoothing and enlargement.

efficiency $(\%)$

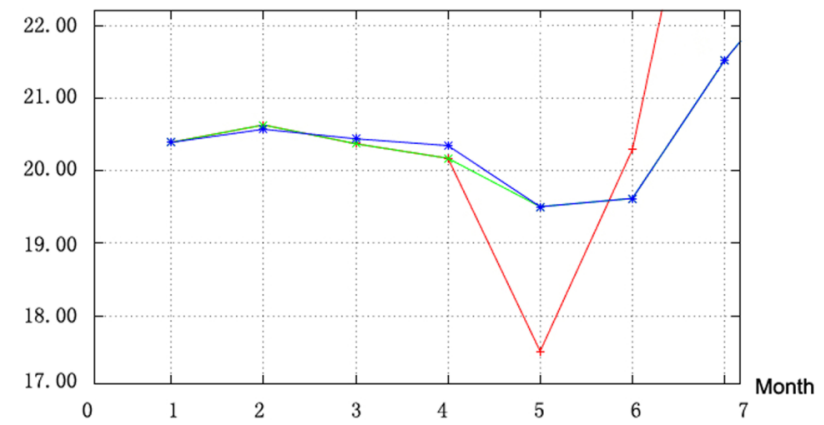

Fig. (4). Comparison of lag time points.

weighted moving average curve based on weight transfer is better than the weighted moving average curve improved, effective improved the hysteresis. In Fig. (5) shows in the coordinate range, the green curve significantly affected by the value of hopping, the predicted values are affected is small, after the improved blue curve value can be ignored outburst effect. 


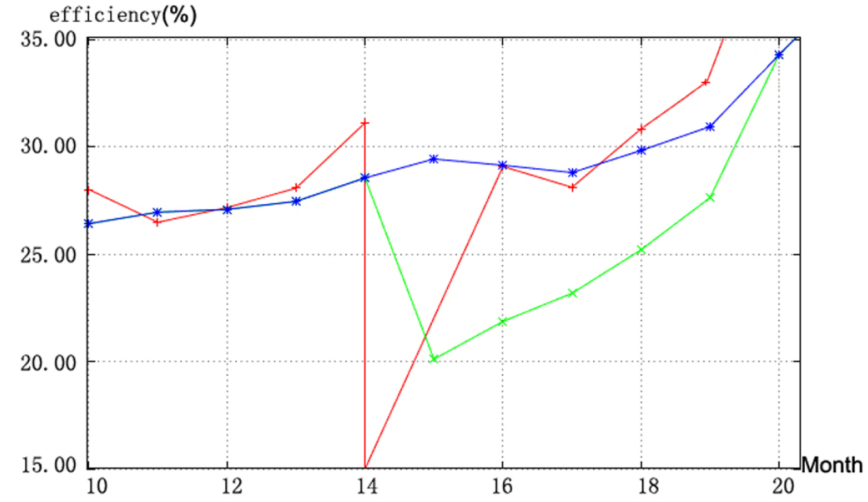

Fig. (5). Comparison of outburst value.

\section{CONCLUSION}

This paper departure from warning research of energyconsuming equipment energy, this paper presents a novel weight transfer method, which fully considered for the lag time, the value of data outburst effects on the overall forecast, then apply weighted moving average of the values of hysteresis and outburst situation improved, the actual application shows that the effect is very obvious improvement.

\section{CONFLICT OF INTEREST}

The authors confirm that this article content has no conflicts of interest.

\section{ACKNOWLEDGEMENT}

Declared none.

\section{REFERENCES}

K. Mehdi, M. Bijari, and G. A. R. Ardali, "Improvement of autoregressive integrated moving average models using fuzzy logic and artificial neural networks (ANNs)", Neurocomputing, vol. 72, no. 4, pp. 956-967, 2009.

[2] H. Q. Han, L. Xie, F. Ding, and X. G. Liu, "Hierarchical leastsquares based iterative identification for multivariable systems with moving average noises", Mathematical and Computer Modelling, vol. 51, no. 9, pp. 1213-1220, 2010.

[3] D. Q. Wang, "Least squares-based recursive and iterative estimation for output error moving average systems using data filtering”, IET Control Theory \& Applications, vol. 5, no. 14, pp. $1648-1657,2011$
[4] Q. Li and Y. X. Chen, "Lag of the Moving Average Method", Statistics and Decision Making, vol. 22, pp. 152-153, 2008.

[5] X. Y. Gong and Z. X. Zhao, "Application of Improved Moving Average Filter to Identification of PSO", Control Engineering of China, vol. 18, no. 4, pp. 556-558, 2011.

[6] D. Joanna, D. Adam, and R. A. Dariusz, "The impact of the modified weighted moving average on the performance of the RED mechanism", Computer Networks, Springer Berlin Heidelberg, pp. 37-44, 2011

[7] D. A. Cook, M. Coory, and R. A. Webster, "Exponentially weighted moving average charts to compare observed and expected values for monitoring risk-adjusted hospital indicators", BMJ quality \& safety, vol. 20, no. 6, pp. 469-474, 2011.

[8] Z. L. Liu, "The Dynamic Analysis of China's Energy-EconomyEnvironment System: VAR and VEC Modeling", Advances in Information Sciences and Service Sciences, vol. 4, no. 14, pp. 210 218, 2012. doi: 10.4156/AISS.vol4.issue14.25

[9] P. Facco, F. Doplicher, F. Bezzo, M. Barolo, "Moving average PLS soft sensor for online product quality estimation in an industrial batch polymerization process", Journal of Process Control, vol. 19 , no. 3 , pp. 520-529, 2009.

[10] H. C. Lee and W. A. Daniel, "Improved design of robust exponentially weighted moving average control charts for autocorrelated processes", Quality and Reliability Engineering International, vol. 27, no. 3, pp. 337-352, 2011.

[11] N. A. Saleh, M. A. Mahmoud, and G. A. S. Abdel-Salam, "The performance of the adaptive exponentially weighted moving average control chart with estimated parameters", Quality and Reliability Engineering International, vol. 29, no. 4, pp.595-606, 2013.

[12] Z. Yang, S. W. Wang, and F. Xiao, "A statistical fault detection and diagnosis method for centrifugal chillers based on exponentially-weighted moving average control charts and support vector regression", Applied Thermal Engineering, vol. 51, no. 1, pp. 560-572, 2013.

(C) Du and Yang; Licensee Bentham Open.

This is an open access article licensed under the terms of the (https://creativecommons.org/licenses/by/4.0/legalcode), which permits unrestricted, non-commercial use, distribution and reproduction in any medium, provided the work is properly cited. 